J. Astrophys. Astr. (xxxx) xx, 000-000

\title{
X-ray flares observed from six young stars located in the region of star clusters NGC 869 and IC 2602
}

\author{
Himali Bhatt ${ }^{1}$, J. C. Pandey ${ }^{2}$, K. P. Singh ${ }^{3}$, Ram Sagar², Brijesh Kumar² \\ ${ }^{1}$ Astrophysical Sciences Division, Bhabha Atomic Research Center, Trombay, Mumbai 400 085, India \\ ${ }^{2}$ Aryabhatta Research Institute of Observational Sciences, Manora Peak, Nainital 263 129, India \\ ${ }^{3}$ Tata Institute of Fundamental Research, Mumbai 400 005, India
}

Received xxx; accepted xxx

\begin{abstract}
.
We present an analysis of seven intense X-ray flares observed from six stars (LAV 796, LAV 1174, SHM2002 3734, 2MASS 02191082+5707324, V553 Car, V557 Car) for the first time. These stars are located in the region of young open star clusters NGC 869 and IC 2602. These flares detected in the XMM-Newton data show a rapid rise (10-40 minutes) and a slow decay (20-90 minutes). The X-ray luminosities during the flares in the energy band $0.3-7.5 \mathrm{keV}$ are in the range of $10^{29.9}$ to $10^{31.7} \mathrm{erg} \mathrm{s}^{-1}$. The strongest flare was observed with the ratio $\sim 13$ for count rates at peak of the flare to the quiescent intensity. The maximum temperature during the flares has been found to be 100 MK. The semi loop lengths for the flaring loops are estimated to be of the order of $10^{10} \mathrm{~cm}$. The physical parameters of the flaring structure, the peak density, pressure, and minimum magnetic field required to confine the plasma have been derived and found to be consistent with flares from pre-main sequence stars in the Orion and the Taurus-Auriga-Perseus region.

Key words: Open clusters and associations:X-rays; stars: premain sequence;stars:Individual(BD+56 526, LAV 796, LAV 1174, SHM2002 3734, V553 Car, V557 Car); stars: X-rays
\end{abstract}

\section{Introduction}

Stellar flares are events in which a large amount of energy is released in a short interval of time. Flares are frequently observed from low mass $(<2$ $\mathrm{M}_{\odot}$ ) active stars throughout the electromagnetic spectrum from radio to X-rays, e.g., $\mathrm{H} \alpha(6563 \AA)$, X-rays $(1.2-124 \AA)$ and radio $(6-20 \mathrm{~cm})$. In a 13 days long observation of Orion (Chandra Orion Ultradeep Project), Favata 
et al. (2005) reported an average of 1.5 flares per star from young solar analogs. In a standard model, stellar flares are known to be a manifestation of the reconnection of magnetic loops, accompanied by particle beams, chromospheric evaporation, rapid bulk flows or mass ejection, and heating of plasma confined in loops (e.g., Sweet 1958, Parker 1957, Petschek 1964, Yokoyama \& Shibata 1997, 1998, 2001, Priest \& Forbes 2002, Haisch 1989, Güdel 2002). The flares produced by pre-main-sequence (PMS) stars are often detected in the X-rays. These X-ray flares show extreme luminosities $\left(\geq 10^{32} \mathrm{erg} \mathrm{s}^{-1}\right)$ and very hot temperatures up to $100 \mathrm{MK}$ (e.g., Skinner et al. 1997; Tsuboi et al. 1998; Imanishi et al. 2002; Feigelson et al. 2002; Skinner et al. 2003; Preibisch et al. 2005). Wolk et al. (2005) studied the properties of flares of PMS stars in the Orion Nebula cluster and reported the median peak luminosity of $10^{30.97} \mathrm{erg} \mathrm{s}^{-1}$ with extremely hard spectra at peak time. An analogous study was presented by Stelzer et al. (2007) for PMS stars in the Taurus Molecular Cloud. The extreme flaring recorded on these PMS stars may have an important bearing on coronal heating. They are therefore providing observational evidence for the type of structures postulated by magnetospheric accretion models for PMS stars. Flares from PMS stars though present many analogies with the solar flares but they also show significant differences, such as the amount of energy released. Therefore, analysis of the light curves during flares can provide insights into the characteristics of the coronal structures and ,therefore, of the magnetic field (Osten et al. 2010, Pandey \& Singh 2008, Reale et al. 2004).

There is little observational evidence of flaring from massive $\left(>10 \mathrm{M}_{\odot}\right)$ and intermediate mass $\left(2-10 \mathrm{M}_{\odot}\right)$ stars. Only a few examples are known: these are $\lambda$ Eri (Smith et al. 1993), MWC 297 (Hamaguchi et al. 2000), $\sigma$ Ori E (Sanz-Forcada et al. 2004), and $\theta^{2}$ Ori A (Petit at el. 2012). The presence of the high temperature plasma with $\mathrm{kT}>2 \mathrm{keV}$ during the flaring activities in massive and intermediate mass stars cannot be explained in the standard framework for understanding X-ray emission from massive stars, i.e., wind shock model (Lucy \& White 1980, Owocki \& Cohen 1999, Kudritzki \& Puls 2000; Crowther 2007; Bhatt et al. 2010a,b), which predict the plasma temperatures of $<1 \mathrm{keV}$. These flares are either explained by magnetically confined wind shock models (Babel \& Montmerle 1997) or associated with nearby companion stars (Petit et al. 2012), but no convincing explanation has been forthcoming.

In this paper, we report evidence of flaring activity in lightcurves of six stars which were detected in a timing analysis of the XMM-Newton observations of eight young open clusters described in our previous paper (Bhatt et al. 2013; hereafter Paper I). The clusters were selected based on their ages ranging from $4 \mathrm{Myr}$ to $46 \mathrm{Myr}$ in order to bridge the gap between very young clusters like the Orion and relatively older clusters like the Pleiades to constrain the time evolution of X-ray emission. In Paper I, we have established the probable cluster membership of the X-ray sources on the basis of 
multi-wavelength properties of the X-ray sources and their location in colormagnitude diagrams of the cluster. Out of the eight young star clusters studied in Paper I, only NGC 869 and IC 2602 star clusters contain all 6 Xray flare stars under present study. They are identified with the massive star BD+56 526 (B1III;Skiff 2009), intermediate mass stars LAV 796 (B7;Currie et al. 2010 (ID\#1413)) and LAV 1174 (B9;Currie et al. 2010 (ID\#376)), and low mass stars SHM2002 3734 (A7;Currie et al. 2010 (ID\#3212)), V553 Car and V557 Car (G0;Glebocki \& Gnacinski 2005), respectively. The basic properties of these sources are given in Table 1, along with relevant information about their host clusters. Though the region of both star clusters NGC 869 and IC 2602 were observed earlier in X-rays by Currie et al. (2010) and by second ROSAT PSPC Catalog 2000 respectively, the timing characteristics of these sources were not studied. In fact, X-ray flare activity from these six sources are reported here for the first time. Their observed V magnitudes and (B-V) colors are given in Table 1. Their photometric spectral types have been derived on the basis of their intrinsic colors after correcting their extinction towards their corresponding clusters, i.e., $(B-V)_{0}=(\mathrm{B}-\mathrm{V})$ $\mathrm{E}(\mathrm{B}-\mathrm{V})$, and given in Table 1 . The spectroscopic information about these sources has been taken from Vizier ${ }^{1}$ services.

The present study provides a valuable sample of X-ray flares from stars in different mass groups to investigate the possible mechanisms for generation of X-ray flares. In addition, the morphologies of these flares are also derived along with a general comparison of the characteristics of these flares with those of other X-ray active stars and the Sun. In the following section, detection of X-ray flares and their possible association with massive, intermediate and PMS low mass stars has been explored. The characteristics of flares are defined in section 3. The physical parameters of coronal loop structures have been modeled in section 4 while results are discussed and concluded in section 5 .

\section{Detection of X-ray flares}

The details of observations, data reduction and identification procedure of the X-ray sources within Two-micron all sky survey (2MASS) Point Source Catalog (PSC; Cutri et al. 2003) and Optical spectroscopic catalogues of stars from Webda ${ }^{2}$ and Vizier ${ }^{3}$ are given in Paper I where each X-ray source detected in data reduction procedure has been ascribed a unique identification number (ID). The IDs of X-ray sources (in Paper I) with flare like features are given in Table 1. The light curves and spectra of the stars

\footnotetext{
${ }^{1}$ http://vizier.u-strasbg.fr/viz-bin/VizieR

${ }^{2}$ http://www.univie.ac.at/webda/navigation.html

${ }^{3}$ http://vizier.u-strasbg.fr/viz-bin/VizieR
} 
within the open clusters have been extracted using circular extraction regions with radii varied between $8^{\prime \prime}$ and $40^{\prime \prime}$ centered on the source position in the energy range of $0.3-7.5 \mathrm{keV}$. The background data have been taken from several neighboring source-free regions on the detectors. Data have been binned from $400 \mathrm{~s}$ to $1600 \mathrm{~s}$. X-ray light curves of stars showing flaring features are shown in Figure 1, where the flare regions have been represented by arrows and marked by $\mathrm{F} i$, where $i=1,2, \ldots, 7$ refers to the flare number (FN), and the quiescent regions have been marked as ' $Q$ '. The probabilities of the variability in the X-ray lightcurves were derived using $\chi^{2}$ test and are given in Figure 1. In the X-ray light curves, flares are characterized by two or more consecutive time bins that constitute a sequence of either rising or falling count rates, corresponding to rise and decay phase of the flare. A rise immediately followed by a decay with a peak count rates greater than $3 \sigma$ of the count rates in quiescent regions ' $Q$ ' is counted as one flare. In this way seven flares ,F1 to F7, were detected. Except from star IC 2602 \# 48, only one flare event was detected from each star.

\subsection{Massive and Intermediate mass stars}

Three flares F4, F1 and F2 are suspected to be related to massive star $\mathrm{BD}+56526$ and intermediate mass stars LAV 796 and LAV 1174, respectively. Therefore, we have carefully examined these X-ray sources to check for the possibility of X-ray emission from presumed nearby/companion star since most of the flare stars have an optical/NIR counterpart within $3^{\prime \prime}$ (see paper I), except BD+56 526. Flare F4 from the X-ray source \# 111 of cluster NGC 869 is detected from a position that has an offset of $6^{\prime \prime}$ from the massive star BD+56 526 .

Because of the large offset between the position of $\mathrm{BD}+56526$ and that of X-ray source \# 111 in NGC 869, the association of X-ray source with the massive star BD+56 526 is doubtful. This X-ray emission could be either from the B-type massive star, BD+56 526, or a presumed T-Tauri star located close to $\mathrm{BD}+56526$ but unresolved in the present observations. In order to check whether the position had shifted between the flaring episode and the quiescent interval, we have extracted X-ray images of the source before and during the flaring activity. These images are shown in Figure 2. During the quiescent state no X-ray emission has been detected within a circle of $6^{\prime \prime}$ radius around the X-ray source position during the flare. This implies that the X-ray emission is dominated by the flare. Further, the optical and 2MASS NIR counterparts of the NGC 869\#111 have been searched within a radius of $6^{\prime \prime}$ about the $\mathrm{X}$-ray source in the available catalogues. No optical detection other than BD+56 526 has been seen with in USNO B-1.0 catalogue down to $\mathrm{V}=21 \mathrm{mag}$. However, an optical counterpart $(\mathrm{V}=19.401$ mag) has been detected with an offset of 2 .' 3 in the recent photometry carried out by Mayne et al. (2007). For convenience, we name this source as NGC 
869\#opt111. This optical position is marked by a cross symbol in Figure 2 . In the 2MASS catalogue, one source, 2MASS 02191082+5707324, has been detected only in the $\mathrm{H}$-band $(\mathrm{H}=15.274 \pm 0.454 \mathrm{mag})$ which is marked by a diamond in Figure 2 with an offset of $1^{\prime \prime}$ from NGC 869\#111. The offset between 2MASS $02191082+5707324$ and the optical counterpart is 1".6. Recently, X-ray data from CHANDRA mission have been published by Currie et al. (2009) for the cluster NGC 869. We have searched for the corresponding counterpart of NGC 869\#111 in the CHANDRA X-ray source catalogue, and found two sources within $6^{\prime \prime}$ search radius. These sources are identified with CHANDra NGC 869\#51 and Chandra NGC 869\#54 (Currie et al. 2009) and marked in Figure 2 using squares. They show an offset of 2 ". 6 and 5."7, respectively, from the X-ray source NGC 869\#111. The offset of the optical and the NIR detection from the CHANDRA NGC 869\#51 are $0 . .4$ and 1".8, respectively. All the sources, namely, NGC 869\#opt111, 2MASS 02191082+5707324 and CHANDRA NGC 869\#51 are located towards the south-west direction of NGC 869\#111 (Figure 2). It appears that the XMM-Newton detection is most likely associated with one of these sources. It is also quite possible that all the four objects identified with optical ( NGC 869\#opt111), 2MASS ( 2MASS 02191082+5707324), Chandra (NGC 869\#51) and XMM-Newton (NGC 869\#111) catalogues, are from a single source as their offsets are very small. It also suggests that the XMM-Newton detection ( NGC 869\#111) is not likely to be associated with the star BD +56 526. Furthermore, the X-ray luminosity of BD+56 526 has been predicted to be of the order of $10^{31} \mathrm{erg} \mathrm{s}^{-1}$ using the relation from Berghöfer et al. (1997) and Sana et al. (2006), whereas the X-ray luminosity during quiescent state is found to be $\sim 10^{30} \mathrm{erg} \mathrm{s}^{-1}$, based on the mean count rate of 0.003 counts $\mathrm{s}^{-1}$ during the quiescent state and the corresponding flux estimated using WebPIMMS ${ }^{4}$ with APEC model for $1 \mathrm{keV}$ plasma. Therefore, we do not believe we have detected X-rays from BD+56 526 directly, and the flare-like feature from the neighbourhood of BD+56 526 is probably associated with a nearby low mass star which is not resolved due to the poor resolution of XMM-Newton.

The photometric spectral type of the source NGC 869\#111 (2MASS $02191082+5707324$ ) has been estimated assuming that it is a member of cluster NGC 869 with optical and NIR counterparts. Using the distance $(2.3 \mathrm{kpc})$ of the cluster NGC 869 and the value of $\mathrm{V}=19.401 \mathrm{mag}$ from Mayne et al. (2007) photometry, the absolute magnitude $\mathrm{M}_{V}$ of XMMNewton source is estimated to be nearly 5.86 mag. The observed (V-H) colour of the XMM-Newton source is found to be nearly 4.13 mag. Using the reddening towards the cluster $\mathrm{E}(\mathrm{V}-\mathrm{H})=1.467 \mathrm{mag}$, the intrinsic color of the XMM-Newton source is derived to be 2.66 mag. The intrinsic $(\mathrm{V}-\mathrm{H})$

\footnotetext{
${ }^{4}$ http://heasarc.gsfc.nasa.gov/Tools/w3pimms.html
} 
color and absolute $\mathrm{V}$ band magnitude are consistent with those for a K-type low mass star (Landolt-Börnstein 1982; Ducati et al. 2001), and at the age of $13 \mathrm{Myr}$, for the cluster NGC 869, this X-ray source could be a low mass PMS star. The uncertainty in the V-band is not given in the catalogue by Mayne et al. (2007), therefore the errors in absolute magnitude and colors cannot be determined. However, the large error in the H-band magnitude produces an error of two to three subclasses in the determination of the spectral type.

Currie et al. (2009) have reported high spatial resolution observations of the open cluster NGC 869 with the CHANDRA X-ray observatory. In their catalogue, X-ray source with identification number \#182 is found to be only $3^{\prime \prime}$ away from our X-ray position of LAV 1174. The offset between its 2MASS NIR position and CHANDRA positions is found to be $0.3^{\prime \prime}$. No other X-ray sources were detected in CHANDRA data within 6 "search radius of LAV 1174, spatial resolution limit of XMM-Newton. Therefore, it is quite likely that the X-ray flare F2 detected by us is associated with X-ray source \#182 with Chandra observations. The X-ray source \#182 in Chandra catalogue was associated with an intermediate mass star \#376 (LAV 1174) in an optical spectroscopic catalogue reported by Currie et al. (2010). No $\mathrm{X}$-ray source has been detected within 6 "search radius of our X-ray position of LAV 796 in the open cluster NGC 869 in ChAndra data. Thus, X-ray flares F1 and F2 appear to be related to intermediate mass stars LAV 796 and LAV 1174, and we are not able to rule out the possibility that X-ray flares arise directly from intermediate mass stars.

\subsection{Low mass stars}

Three X-ray flares F3, F5 and F6 are associated with low mass stars SHM2002 3734, V553 Car and V557 Car, respectively. X-ray emission from SHM2002 3734 was observed by CHANDRA from a source with an identification number \# 255 in Currie et al. (2009) catalogue and has an offset of only $3^{\prime \prime}$ with our X-ray positions. Two X-ray sources J104100.6-642003 and J104241.3642106 with an offset of about $3^{\prime \prime}$ from V553 Car and V557 Car, respectively have been reported in the second ROSAT PSPC Catalog (2000). These two sources V553 Car and V557 Car in the open cluster IC 2602 are probably responsible for the three flares F5, F6 and F7 and characterised as BY Dratype variables in the 76th name list of variable stars (Kazarovets, Samus \& Durlevich 2001)" 
X-ray Flares from young stars in the open clusters NGC 869 and IC 26027

\section{X-ray flare characteristics}

\subsection{Rise time and Decay time}

The lightcurves of the flares (see Figure 1) are characterised by a fast rising phase followed by a slower decay phase, which are similar to the solar flares and thus solar-like magnetic reconnection events which govern by conduction and radiation cooling mechanisms. This fast-rise exponential decay shape (usually called FRED) are commonly associated with stellar flares from PMS stars and fitted with exponential function (see Stelzer, Neuhäuser \& Hambaryan 2000, Güdel 2004 ). The e-folding rise and decay times have been determined from the least-squares fit of the exponential function

$$
\mathrm{c}(\mathrm{t})=\mathrm{A}_{0} \exp ^{-\left[\left(\mathrm{t}-\mathrm{t}_{0}\right) / \tau_{\mathrm{r}, \mathrm{d}}\right]}+\mathrm{q}
$$

where $c(t)$ is the count rate as a function of time $t, t_{0}$ is the time of peak count rate, $q$ is the count rate in the quiescent state, $\tau_{d} / \tau_{r}$ is the decay/rise time of the flare and $A_{0}$ is the count rate at flare peak. The quiescent state count rates were taken as mean value of count rates during the 'Q' in the light curves. The least-square fits of the eq. 1 are shown in Figure 1 by solid lines. The values of $\tau_{d}$ and $\tau_{r}$ along with the start time, flare duration, quiescent state count rates and ratio of count rate at flare and quiescent state are given in Table 2 for all the seven flares observed. The rise time of these flares were found in the range of 10-40 minutes, whereas the e-folding decay times were found in the range of 20-90 minutes. The longest duration $(\sim 25 \mathrm{ks})$ flare was observed from the star \# 42 of NGC 869 (SHM2002 3734). The peak flare count rates were found to be 1.5-13.3 times more than that found during quiescent state. The strongest flare was observed from the star \# 111 of NGC 869 (2MASS 02191082+5707324), where the ratio of peak to the quiescent count rate was found to be 13.3 .

\section{$3.2 \quad X$-ray spectra of flares}

X-ray spectra of the flares have been analysed to look for the spectral evolution during the flares. Spectral analysis has been performed based on global fitting using the Astrophysical Plasma Emission Code (APEC) version 1.10 modeled by Smith et al. (2001) and implemented in the XSPEC version 12.3.0. The absorption toward the stars by interstellar medium was accounted for by using multiplicative model photoelectric absorption screens (PHABS) in XSPEC (Balucińska-Church \& McCammon 1992). In order to study the flaring component only, we have performed "2T APEC" model spectral fits of the data during the flare state. The quiescent emission taken into account by including its best-fitting $1 \mathrm{~T}$ model as a frozen background contribution. This is equivalent to considering the flare emission after subtraction of the quiescent level, and allows us to derive the effective average temperature 
and the emission measure (EM) of the flaring plasma. The spectral parameters and coronal temperatures for quiescent state (Q) are derived in paper I, and the values of hydrogen column density $\left(N_{H}\right)$ and abundance $(Z)$ parameters have been kept fixed to the quiescent state values in the spectral fitting (see Table 3). The best-fit parameters during the flare are given in Table 3. The X-ray luminosity during the flares varied from $10^{29.9}$ to $10^{31.7}$ $\mathrm{erg} \mathrm{s}^{-1}$. For the flare F4 from LAV 796, the X-ray luminosity during the flare was found to be $10^{31.7}$, which is 16.6 times more than that found during the quiescent state. According to Reale (2007), a typical output of the analysis of X-ray spectra with moderate resolution, such as those from CCD detectors (e.g. EPIC/XMM-Newton), is the average temperature of the flaring loops which is usually lower than the real loop maximum temperatures. The loop maximum temperature for the EPIC instruments are calibrated as $\mathrm{T}_{\max }=0.13 \mathrm{~T}_{\text {obs }}^{1.16}$ (Reale 2007).

\section{Modeling the X-ray flares based on coronal loops}

The characteristics of these flares are all consistent with the flares from low mass PMS stars in young open clusters, e.g., the Orion (Getman et al. 2008a), and therefore, can be associated with the presence of a magnetically confined corona and thus of an operational dynamo mechanism. The physical sizes and morphology of the loop structures involved in a stellar flare can be estimated using flare loop models by an analogy with solar flares. However, it has been considered that flares occur inside closed coronal loops anchored to the photosphere, plasma is confined inside each loop, and its bulk motion and energy transport occur only along the magnetic field lines ( Reale et al. 1997).

A method to infer the geometrical sizes and other relevant physical parameters of the flaring loops was presented by Reale et al. (1997), which was based on the decay time, and on evolution of temperature and the EM during the flare decay. However, this method needs time resolved spectroscopy, therefore, could not be used for the present analysis. Haisch (1983) assumed that $\tau_{\mathrm{d}}$ is comparable to the radiative and conductive cooling times, and estimated the loop length $\left(\mathrm{L}_{\mathrm{Ha}}\right)$ as,

$$
\mathrm{L}_{\mathrm{Ha}}=5 \times 10^{-6} \mathrm{EM}^{0.25} \tau_{\mathrm{d}}^{0.75}
$$

Hawley et al. (1995) described an approach, which is based on rise and decay times. In this approach, strong evaporative heating is dominant during the rising phase, while $\mathrm{X}$-ray emission is dominated by radiative cooling during

the decay phase. At the loop top, there is an equilibrium and the loop length can be derived as

$$
\mathrm{L}_{\mathrm{r}}=\frac{1500}{\left(1-\mathrm{x}_{\mathrm{d}}^{1.58}\right)^{4 / 7}} \cdot \tau_{\mathrm{d}}^{4 / 7} \cdot \tau_{\mathrm{r}}^{3 / 7} \cdot\left(\mathrm{T}_{\max }\right)^{1 / 2}
$$


where $T_{\max }$ is temperature at flare apex and $x_{d}^{2}=q / A_{0}$. We have sufficient information to model a loop by rise and decay method and Haisch approach. The loop-lengths of the flares were derived in the rage of $1.3-3.8 \times 10^{10} \mathrm{~cm}$ using the rise and decay method, while it was $1.8-9.8 \times 10^{10} \mathrm{~cm}$ using Haisch approach. In general, the loop lengths derived from Haisch approach are found to be more than that derived from rise and decay method. However, in most of the cases the differences between loop lengths derived from these two methods are well with $1 \sigma$ level. The flare F3 from the star SHM2002 3734 in the open cluster NGC 869 is only the flare where the difference between loop lengths derived from two methods is more than $2 \sigma$ level.

After knowing loop lengths, the various loop parameters like maximum pressure, plasma density, loop volume and minimum magnetic field to confine the plasma can be derived. The analysis of X-ray spectra provides values of the temperature and EM. From the EM and the plasma density $\left(n_{e}\right)$, the volume $(\mathrm{V})$ of the flaring loop is estimated as $E M=n_{e}^{2} \mathrm{~V}$. Using the loop scaling laws, the maximum pressure, temperature, loop length and heating rate per unit volume are related as (Rosner, Tucker \& Vaiana 1978, Kuin \& Martens 1982):

$$
\begin{gathered}
T_{\max }=1.4 \times 10^{3}(p L)^{1 / 3} \\
E_{H} \approx 10^{-6} T_{\max }^{3.5} L^{-2} \text { ergs } \mathrm{s}^{-1} \mathrm{~cm}^{-3}
\end{gathered}
$$

The minimum magnetic field necessary to confine the flaring plasma can be estimated as $B=\sqrt{8 \pi p}$.

The derived parameters are given in Table 4. Using the equation 4 , the maximum pressure was found to be in the range of $5 \times 10^{2}-8.75 \times 10^{3}$ dyne $\mathrm{cm}^{-2}$ during the flare. Assuming that the hydrogen plasma is totally ionized, the maximum plasma density in loop during the flare was found from $5 \times 10^{10}$ to $1.5 \times 10^{12} \mathrm{~cm}^{-3}$. Using the observed EM, the loop volume was computed in the range of $1.5 \times 10^{30}-5.7 \times 10^{32} \mathrm{~cm}^{3}$. A hint for the heat pulse intensity comes from the flare maximum temperature. By applying the loop scaling laws (see eq. 5) and loop maximum temperature (see Table 4 ), the heating rates per unit volume $\left(E_{h}\right)$ are found from 0.3 to $92 \mathrm{erg} \mathrm{s}^{-1}$ $\mathrm{cm}^{-3}$. From the derived pressure of the flare plasma, minimum magnetic field required to confine the plasma are found to be in the range of 0.1-1.5 kilo Gauss.

\section{Discussion and Conclusions}

We have carried out analysis of seven flares observed from six stars located in the region of young star clusters NGC 869 and IC 2602. Out of six stars, four have been classified as low mass stars, while two stars LAV 796 and LAV 1174 have been classified as intermediate mass stars. The strongest flare 
was observed from the star \# 111 of NGC 869 with a ratio of peak flare to quiescent state count rates of $\sim 13$. It is detected from the neighbourhood of $\mathrm{BD}+56526$ and is probably associated with a nearby low mass star, 2MASS $02191082+5707324$, which is not resolved due to the poor resolution of XMM-Newton.

The rise and decay times of the flares reported here are lower than that of the flares from PMS stars in Orion (between 1 hour to 1 day;Getman et al. 2008a), but, compared to that of solar flares (e.g., only a few minutes; Priest \& Forbes 2002), PMS stars of Pleiades cluster (Stelzer, Neuhäuser \& Hambaryan 2000) and main-sequence (MS) stars (Pandey \& Singh 2008). The peak to quiescent state count rate ratio for these flares are in the range of 1.5 to 13.3. The maximum flare plasma temperatures are found to be comparable to the flares from PMS, MS and RCVn-type stars (e.g., Pandey \& Singh 2008, 2012; Getman et al. 2008b; Favata et al. 2005). The total energy released during the X-ray flares $\left(=\mathrm{L}_{\mathrm{X}} \times\right.$ duration of flare $)$, has been found to be in the range of $10^{34.0}-10^{35.8}$ ergs. This indicates that these flares are $10^{4-6}$ times more energetic than the solar flares (Moore et al. 1980), but similar to the flares observed from field dwarfs, and evolved RS CVn-type binaries (e.g., Pandey \& Singh 2008, 2012). The inferred sizes of the flaring structures are consistent with the results presented by Getman et al. (2008b) and Favata et al. (2005) for certain PMS stars. However, the derived values of the maximum plasma temperatures and loop lengths are larger than that of the solar flares. The giant X-ray emitting arches found in the Sun are to have typical sizes of $10^{8} \mathrm{~cm}$ with the maximum temperature of 10 MK (e.g., Getman \& Livshitz 1999;Getman \& Livshits 2000). These parameters are similar to the parameters derived for the PMS stars in Taurus-Auriga-Perseus region (Stelzer, Neuhäuser \& Hambaryan 2000) and the Orion (Getman et al. 2008b; Favata et al. 2005).

X-ray luminosities $\left(\mathrm{L}_{\mathrm{X}}\right)$ during the flares from low mass stars SHM2002 3734 (\# 42) in the cluster NGC 869, and V553 Car and V557 Car in the cluster IC 2602 have been found to be of the order of $10^{30-31} \mathrm{erg} \mathrm{s}^{-1}$. It indicates that these flares are more powerful than those observed from field dwarf stars (Pandey \& Singh 2008) but are equally powerful as the flares from PMS stars (Getman et al. 2008b). The loop length of the order of $10^{10} \mathrm{~cm}$ is indicating large coronal structures. For these flares, the estimated maximum electron densities under the assumption of a totally ionized hydrogen plasma have been found in the order of $10^{10-12} \mathrm{~cm}^{-3}$. It is comparable to the values expected from a plasma in coronal conditions (Landini et al. 1986). The strength of the magnetic field (of the order of a few hundred $G$ ), loop sizes of the large-scale magnetic field and X-ray luminosities of the flares suggest that these flares are enhanced analogs of eruptive solar flares. Therefore, these flares can be interpreted as scaled-up versions of solar flares and the flares from PMS stars in the Orion and the Taurus-Auriga-Perseus region.

The loop lengths of the flaring structure from intermediate mass stars 
LAV 796 and LAV 1174 in the cluster NGC 869 have been found to be comparable with that of flares from low mass stars in the present sample and also in other studies (e.g., Getman et al. 2008b; Favata et al. 2005), but larger than that of the solar flares (e.g., Getman \& Livshitz 1999; Getman $\&$ Livshits 2000). However, these values of loop lengths are lower that of the flares from young massive stars MWC 297 ( $10^{11} \mathrm{~cm}$; Hamaguchi et al. 2000) and HD 261902 (10 ${ }^{12} \mathrm{~cm}$;Yanagida et al. 2005). Similarities between the nature of X-ray flares from intermediate mass stars and low mass stars suggest that X-rays from intermediate mass stars are probably arising from unresolved nearby low mass stars (Stelzer et al. 2006, Joshi et al. 2008 and references therein). Recently, Balona (2013) has studied 875 A-type stars using Kepler data in optical band, and reported that variation in light could be due to rotation modulation caused by star-spots. If A-type stars have spots, then it is natural to expect a magnetic field, and therefore the possibility of X-ray activity and magnetic reconnection in intermediate mass stars. If the X-ray emission indeed arises from the intermediate mass stars rather than a hidden low mass star through coronal loop structures then the origin of the magnetic field of the order of 1000 Gauss is hard to explain in the intermediate mass stars which are thought to be fully or nearly-fully radiative.

\section{Acknowledgments}

Authors are thankful to anonymous Referee for providing constructive comments which improved content of the manuscript. This publication makes use of data from the Two Micron All Sky Survey, which is a joint project of the University of Massachusetts and the Infrared Processing and Analysis Center/California Institute of Technology, funded by the National Aeronautics and Space Administration and the National Science Foundation, and data products from XMM-Newton archives using the high energy astrophysics science archive research center which is established at Goddard by NASA. We acknowledge XMM-Newton Help Desk for their remarkable support in X-ray data analysis. Data from Simbad, VizieR catalogue access tool, CDS, Strasbourg, France have also been used. HB is thankful for the financial support for this work through the INSPIRE faculty fellowship granted by the Department of Science \& Technology India, and R. C. Rannot, Nilesh Chouhan and R. Koul for their support to finish this project. 


\section{References}

Babel J., Montmerle T., 1997, A\&A, 323, 121

Balucińska-Church M., McCammon D.,1992,ApJ,400,699

Berghöfer T. W., Schmitt J. H. M. M., Danner R., Cassinelli J. P.,1997,A\&A,322,167

Balona L. A., 2013, MNRAS, 431,2240

Bhatt H., Pandey J. C., Kumar B., Singh K. P., Sagar Ram, 2010a, MNRAS, 402,1767

Bhatt H., Pandey J. C., Kumar B., Sagar R., Singh K. P., 2010b, New Astronomy, 15,755

Bhatt H., Pandey J. C., Singh K. P., Sagar Ram, B. Kumar, 2013, Journal of Astrophysics \& Astronomy, 34, 393, arXiv1311.3025, PAPER I

Currie T., Evans N. R., Spitzbart B. D., Irwin J., Wolk S. J., Hernandez J., Kenyon S. J., Pasachoff J. M.,2009,AJ,137,3210

Currie T., Hernandez J., Irwin J., Kenyon S. J., Tokarz S., Balog Z., Bragg A., Berlind P., Calkins M., 2010, ApJS, 186, 191

Cutri R. M., Skrutskie M. F., van Dyk S., Beichman C. A., Carpenter J. M., Chester T., Cambresy L., Evans T. et al., 2003, The IRSA 2MASS All-Sky Point Source Catalog, NASA/IPAC Infrared Science Archive http://irsa.ipac.caltech.edu/applications/Gator/

Ducati J. R., Bevilacqua C. M., Rembold S. B., Ribeiro D.,2001,ApJ,558,309

Favata F., Flaccomio E., Reale F., Micela G., Sciortino S., Shang H., Stassun K. G., Feigelson E. D., 2005, ApJS, 160, 469

Feigelson E. D., Broos P., Gaffney J. A. III, Garmire G., Hillenbrand L. A., Pravdo S. H., Townsley L., Tsuboi Y.,2002,ApJ,574,258

Getman K. V., Feigelson E. D., Broos P. S., Micela G., Garmire G. P.,2008a,ApJ,688,418

Getman K. V., Feigelson E. D., Micela G., Jardine M. M., Gregory S. G., Garmire G. P.,2008b,ApJ,688,437

Getman K. V., Livshits M. A.,2000, ARep, 44, 255

Getman K. V., Livshitz M. A.,1999, ARep, 43, 615

Glebocki R., Gnacinski P., 2005, Catalog of Stellar Rotational Velocities, 3244

Güdel M., 2002, ARAA, 40, 217

Güdel M., 2004, AAR, 12, 71

Haisch B. M. 1983, in IAU Colloq. 71, Activity in Red-Dwarf Stars, Vol. 102, ed. P. B. Byrne \& M. Rodono (Dordrecht: Reidel), 255

Haisch B.M., 1989, Solar Phys., 121, 3

Hamaguchi K., Terada H., Bamba A., Koyama K., 2000, ApJ, 532, 1111

Hamaguchi K., Terada H., Bamba A., Koyama K., 2000, ApJ, 532, 1111

Hawley S. L., 1995, ApJ, 453, 464

Imanishi K., Nakajima H., Tsujimoto M., Koyama K., Tsuboi, Y., 2003,PASJ, 55, 653

Joshi H., Kumar B., Singh K. P., Sagar R., Sharma S., Pandey, J. C., 2008, MNRAS, 391, 1279

Kazarovets E. V., Samus N. N., Durlevich O. V., 2001, Information Bulletin on Variable Stars, 5135, 1

Kuin N. P. M., Martens P. C. H., 1982, A\&A, 108, 1 
X-ray Flares from young stars in the open clusters NGC 869 and IC 260213

Kudritzki R. P., Puls J., 2000, ARA\&A, 38, 613

Landini M., Monsignori F. B. C., Pallavicini R., Piro L., 1986, A\&A, 157, 217

Landolt-Börnstein, 1982, Numerical Data and Functional Relationship in Science and Technology, New series, Group VI, Vol. 2b, Scaifers K. \& Voigt H. H. (eds.) Springer - Verlog, Berlin

Lucy L. B., White R. L., 1980, ApJ, 241, 300

Mayne N. J., Naylor T., Littlefair S. P., Saunders E. S., Jeffries R. D.,2007, MNRAS,375,1220; VizieR On-line Data Catalog: J/MNRAS/375/1220

Messina, S., Guinan, E. F., 2003, A\&A, 409, 1017

Moore R., McKenzie D. L., Svestka Z., Widing K. G., Dere K. P., Antiochos S. K., Dodson-Prince H. W., Hiei E., Krall K. R., Krieger A. S., 1980, Solar flares: A monograph from Skylab Solar Workshop II (A80-37026 15-92), 341-409

Osten R. A., et al. 2010, ApJ, 721, 785

Owocki S. P., Cohen D. H., 1999, ApJ, 520, 833

Pandey J. C., Singh K. P., 2008, MNRAS, 387, 1627

Pandey J. C., Singh K. P., 2012, MNRAS, 419, 1219

Parker E.N., 1955, ApJ, 121, 491507.

Petit V., Gagne M., Cohen D. H., Townsend R. H. D., Leutenegger M. A., Savoy M. R., Fehon G., Cartagena C. A., Proceedings from ASP Conference "Circumstellar Dynamics at High Resolution", ed. by A. Carciofi and Th. Rivinius

Petschek H.E., 1964, "Magnetic Field Annihilation", in The Physics of Solar Flares, Proceedings of the AAS-NASA Symposium held 28-30 October, 1963 at the Goddard Space Flight Center, Greenbelt, MD, (Ed.) Hess, W.N., vol. SP50 of NASA Special Publications, pp. 425439, NASA Science and Technical Information Division, Washington, DC.

Priest E. R., Forbes T. G., 2002, A\&ARv, 10, 313

Reale F., 2007, A\&A, 471, 271

Reale F., Betta R., Peres G., Serio S., McTiernan J., 1997, A\&A, 325, 782

Reale F., Güdel M., Peres G., Audard M., 2004, A\&A, 416, 733

Rosner R., Tucker W. H., Vaiana G. S., 1978, ApJ, 220, 643

Sana H., Rauw G., Nazé Y., Gosset E., Vreux J.-M., 2006, MNRAS, 372, 661

Sanz-Forcada J., Franciosini E., Pallavicini R., 2004, A\&A, 421, 715

Skiff, B. A. 2009, Catalogue of Stellar Spectral Classifications, VizieR Catalog B/mk/mktypes (Lowell Observatory), 1, 2023

Skinner S., Gagné M., Belzer E., 2003, ApJ, 598, 375

Skinner S. L., Gudel M., Koyama K., Yamauchi S. 1997, ApJ, 486, 886

Slesnick C. L., Hillenbrand L. A., Massey P.,2002,ApJ,576,880

Smith M. A., Grady C. A., Peters G. J., Feigelson E. D., 1993, ApJ, 409, L49

Smith M. A., Grady C. A., Peters G. J., Feigelson E. D., 1993, ApJ, 409, L49

Stelzer B., Flaccomio E., Briggs K., Micela G., Scelsi L., Audard M., Pillitteri I., Güdel M., 2007, A\&A, 468, 463

Stelzer B., Huélamo N., Micela G., Hubrig S., 2006,A\&A,452,1001

Stelzer B., Neuhäuser R., Hambaryan V., 2000,A\&A,356,949

Sweet P.A., 1958, "The neutral point theory of solar flares", in Electromagnetic Phenomena in Cosmical Physics, Proceedings of IAU Symposium No. 6, held in Stockholm, August 27-31, 1956, (Ed.) Lehnert, B., vol. 6 of IAU Symposium, pp. 123134, Cambridge University Press, Cambridge; New York.

Tsuboi Y., Koyama K., Murakami H., Hayashi M., Skinner S., Ueno, S., 1998, ApJ, 
503,894

Wolk S. J., Harnden F. R. Jr., Flaccomio E., Micela G., Favata F., Shang H., Feigelson E. D., 2005, ApJS, 160, 423

Yanagida T., Ezoe Y., Kawaharada M., Kokubun M., Makishima K., 2005, in Protostars and Planets V, p. 8276

Yokoyama, T. and Shibata, K., 1997, ApJL, 474, L61

Yokoyama, T. and Shibata, K., 1998, ApJL, 494, L113

Yokoyama, T. and Shibata, K., 2001, ApJ, 549, 1160 

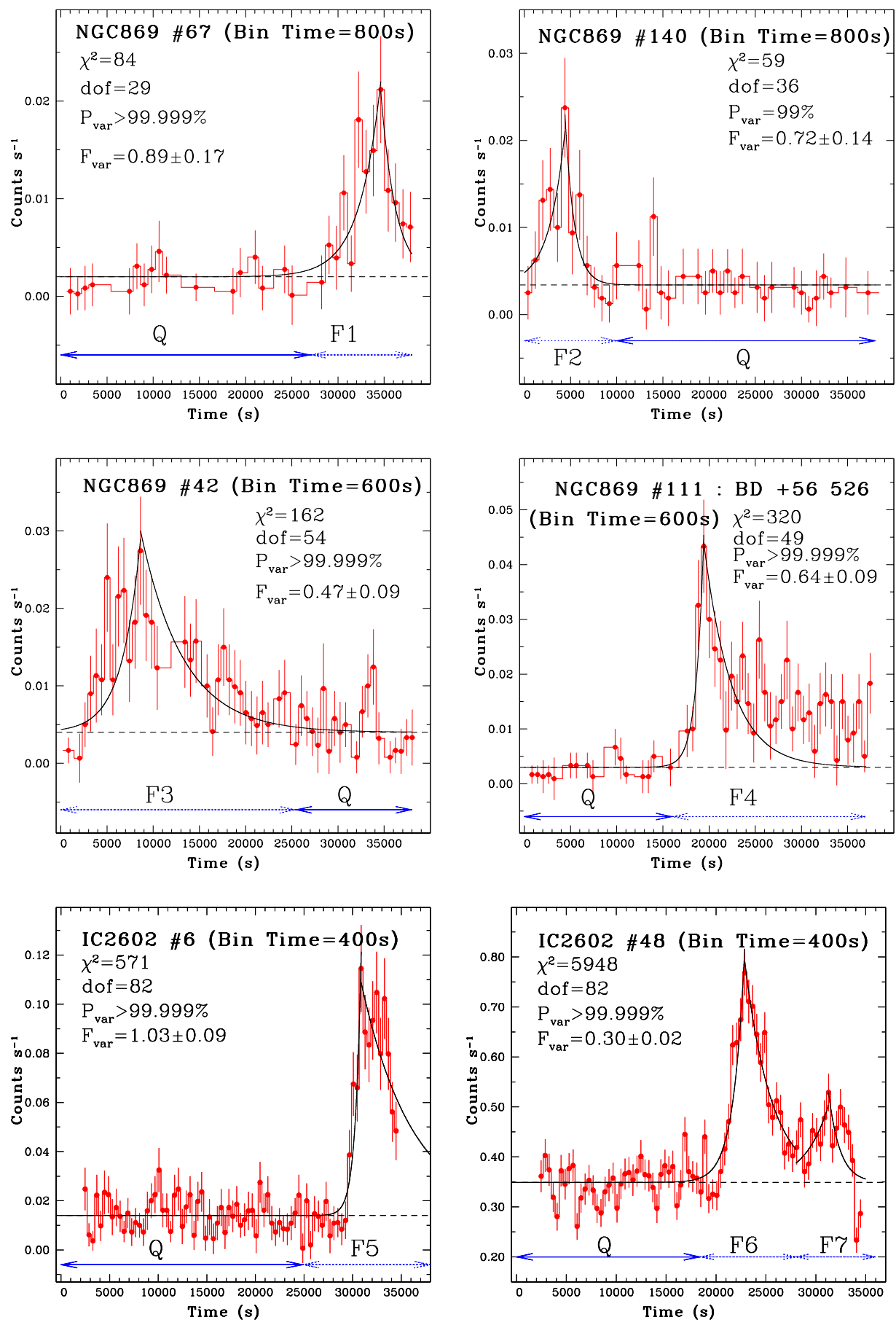

Figure 1 X-ray flares from the cluster members. Flare time intervals are represented by dotted arrows with lines and marked by $\mathrm{F} i$, where $i=1,2, \ldots, 5$ refers to the flare number. Quiescent state ' $Q$ ' time intervals are shown by solid arrows with lines and quiescent state mean count rates are marked with dashed lines. Exponential rise and decay fit are shown by solid lines. 


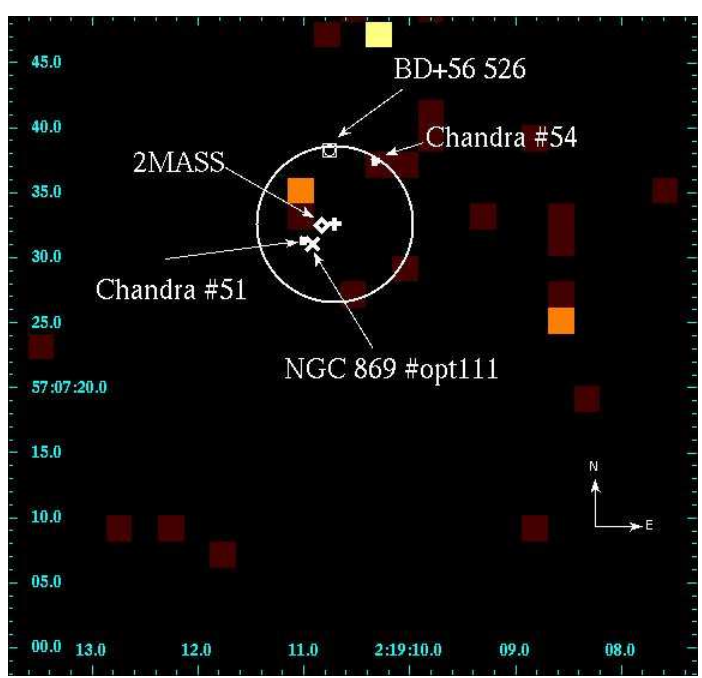

(a)

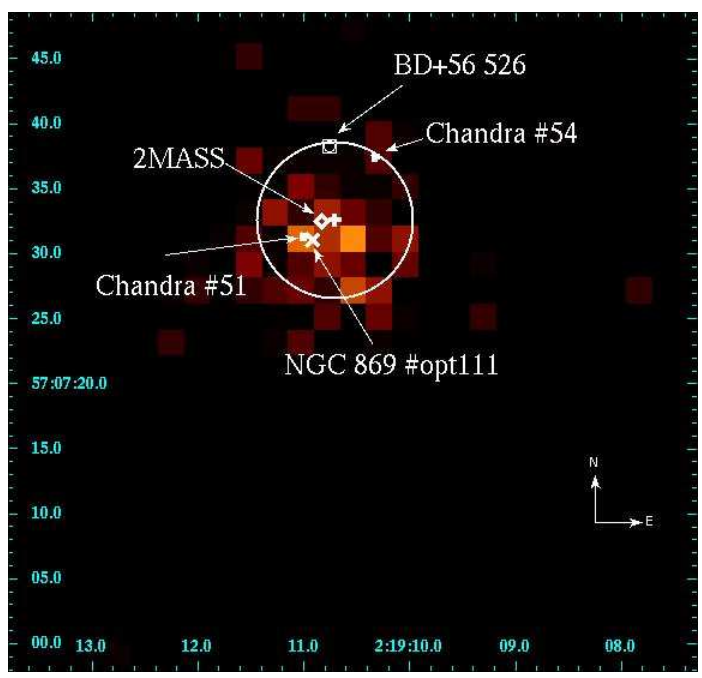

(b)

Figure 2 X-ray image of the field BD+56 526 (a) before flaring event and (b) during the flaring event. $\mathrm{X}$-axis and $\mathrm{Y}$-axis are representing $\mathrm{RA}(\mathrm{J} 2000)$ and DEC(J2000), respectively. The XMM-NEWTON detection position is marked by the symbol of plus. The symbol of the square represents CHANDRA source detection position of source ID \#51 and \#54 from Currie et al. (2009), symbol of diamond represents 2MASS H-band detection position and square with circle represent the position of massive star BD+56 526. The optical position given by Mayne et al. (2007) is marked by the symbol of the cross. 
$X$-ray Flares from young stars in the open clusters NGC 869 and IC 260217

Table 1 Basic properties of stars with flare-like features.

\begin{tabular}{ccccccccc}
\hline Cluster \#ID & Name & $\begin{array}{c}R A_{J 2000} \\
(\mathrm{deg})\end{array}$ & $\begin{array}{c}D E C_{J 2000} \\
(\mathrm{deg})\end{array}$ & $\begin{array}{c}\mathrm{V} \\
(\mathrm{mag})\end{array}$ & $\begin{array}{c}(\mathrm{B}-\mathrm{V}) \\
(\mathrm{mag})\end{array}$ & $\begin{array}{c}(B-V)_{0}^{1} \\
(\mathrm{mag})\end{array}$ & $\begin{array}{c}\text { Spectral type } \\
\mathrm{Ph}^{2}\end{array}$ & $\mathrm{Sp}^{3}$ \\
\hline NGC 869 \# 67 & LAV 796 & 34.662083 & 57.220444 & $14.145^{4}$ & $0.445^{4}$ & $-0.105^{4}$ & $\mathrm{~B} 8$ & $\mathrm{~B} 7^{6}$ \\
NGC 869 \# 140 & LAV 1174 & 34.870708 & 57.163887 & $11.904^{4}$ & $0.407^{4}$ & $-0.143^{4}$ & $\mathrm{~B} 6$ & $\mathrm{~B} 9^{6}$ \\
NGC 869 \# 42 & SHM2002 3734 & 34.586708 & 57.174110 & $15.733^{4}$ & $0.750^{4}$ & $0.220^{4}$ & $\mathrm{~A} 7$ & $\mathrm{~A}^{6}$ \\
NGC 869 \# 111 & BD+56 526 & 34.794624 & 57.125721 & & & & & \\
IC 2602 \# 6 & V553 Car & 160.249878 & -64.334000 & $15.39^{5}$ & $1.570^{5}$ & $1.535^{5}$ & $\mathrm{M} 4$ & \\
IC 2602 \# 48 & V557 Car & 160.72791 & -64.351387 & $10.52^{5}$ & $0.600^{5}$ & $0.565^{5}$ & $\mathrm{G} 0$ & $\mathrm{G0}^{7}$ \\
\hline
\end{tabular}

$\dagger^{\dagger}$ : The identification number (ID) is taken from Paper I which has been assigned to each X-ray source detected during data reduction procedure

1 : Intrinsic colors are estimated from observed colors corrected for the extinction $(\mathrm{E}(\mathrm{B}-\mathrm{V}))$ for their corresponding clusters. For the open clusters NGC 869 and IC 2602, the E(B-V) are taken as 0.55 and $0.035 \mathrm{mag}$, respectively, from Paper I

2 : Spectral type derived from photometric information in the present study

3 : Spectral type derived from spectroscopic studies

4 : Data taken from Slesnick, Hillenbrand \& Massey (2002)

5 : Data taken from Messina \& Guinan (2003)

6 : Spectral type derived from spectroscopic study by Currie et al. (2010)

7 : Spectral type derived from spectroscopic study by Glebocki \& Gnacinski (2005)

* : Most probably a companion/nearby low mass star to this star.

Table 2 Characteristics of flares obtained from the fitting of light curves in energy band $0.3-7.5 \mathrm{keV}$. FN, $\tau_{r}, \tau_{d}$, q and A0/q represent flare number, rising time, decay time, count rates at quescient time interval and ratio of peak count rates and count rates at quescient time interval, respectively.

\begin{tabular}{|c|c|c|c|c|c|c|c|}
\hline Cluster\#ID & FN & $\begin{array}{c}\text { Start Time } \\
\text { ks }\end{array}$ & $\begin{array}{c}\text { Duration } \\
\text { ks }\end{array}$ & $\begin{array}{l}\tau_{r} \\
\mathrm{ks}\end{array}$ & $\begin{array}{l}\tau_{d} \\
\mathrm{ks}\end{array}$ & $\begin{array}{c}\mathrm{q} \\
\operatorname{cts~s}^{-1}\end{array}$ & $\mathrm{~A} 0 / \mathrm{q}$ \\
\hline \multicolumn{8}{|c|}{ Intermediate mass stars } \\
\hline NGC $869 \# 67$ & F1 & 27.0 & 11.0 & $2.33 \pm 0.49$ & $1.60 \pm 0.38$ & $0.002 \pm 0.001$ & 10 \\
\hline NGC $869 \# 140$ & $\mathrm{~F} 2$ & 0.0 & 10.0 & $1.74 \pm 0.75$ & $1.00 \pm 0.31$ & $0.003 \pm 0.002$ & 7 \\
\hline \multicolumn{8}{|c|}{ Low mass stars } \\
\hline NGC 869 \#42 & F3 & 0.0 & 25.0 & $2.03 \pm 0.72$ & $4.62 \pm 0.59$ & $0.004 \pm 0.003$ & 6.2 \\
\hline NGC $869 \# 111$ & F4 & 16.0 & 21.0 & $0.83 \pm 0.14$ & $3.00 \pm 0.86$ & $0.003 \pm 0.002$ & 13.3 \\
\hline IC 2602 \#6 & F5 & 24.8 & 13.2 & $0.59 \pm 0.12$ & $5.28 \pm 1.70$ & $0.014 \pm 0.007$ & 7.8 \\
\hline IC $2602 \# 48$ & F6 & 18.4 & 9.6 & $1.13 \pm 0.17$ & $2.70 \pm 0.25$ & $0.349 \pm 0.036$ & 2.2 \\
\hline IC $2602 \# 48$ & F7 & 28.0 & 8.0 & $2.34 \pm 1.08$ & $1.14 \pm 0.87$ & $0.349 \pm 0.036$ & 1.5 \\
\hline
\end{tabular}


Table 3 Spectral parameters of the X-ray flares and quiescent state.

\begin{tabular}{|c|c|c|c|c|c|c|c|}
\hline \multirow[t]{2}{*}{ Cluster ID } & \multirow[t]{2}{*}{ FN } & \multicolumn{3}{|c|}{ Flare } & \multicolumn{3}{|c|}{ Quiescent State } \\
\hline & & $\begin{array}{c}\mathrm{kT} \\
(\mathrm{keV})\end{array}$ & $\begin{array}{c}\log (\mathrm{EM})) \\
\left(\mathrm{cm}^{-3}\right)\end{array}$ & $\begin{array}{c}\log \left(L_{X}\right) \\
\operatorname{erg~s}^{-1}\end{array}$ & $\begin{array}{c}\mathrm{kT} \\
(\mathrm{keV})\end{array}$ & $\begin{array}{c}\log (\mathrm{EM})) \\
\left(\mathrm{cm}^{-3}\right)\end{array}$ & $\begin{array}{c}\log \left(L_{X}\right) \\
e^{-r g ~ s}{ }^{-1}\end{array}$ \\
\hline NGC869 \#67 & F1 & $6.50_{-2.70}^{+23.90}$ & $54.56_{-0.08}^{+0.08}$ & 31.70 & $0.54_{-0.33}^{+0.43}$ & $53.42_{-0.32}^{+0.56}$ & 30.48 \\
\hline NGC869 \#140 & F2 & $>1.88$ & $54.34_{-0.16}^{+0.13}$ & 31.60 & $1.27_{-0.23}^{+0.43}$ & $53.98_{-0.15}^{+0.13}$ & 31.04 \\
\hline NGC869 \#42 & F3 & $1.55_{-0.55}^{+1.81}$ & $54.18_{-0.21}^{+0.12}$ & 31.62 & $3.66_{-1.90}^{+14.00}$ & $54.25_{-0.12}^{+0.11}$ & 31.38 \\
\hline NGC869 \#111 & F4 & $7.43_{-2.65}^{+8.07}$ & $54.47_{-0.05}^{+0.05}$ & 31.66 & & & $30.00^{1}$ \\
\hline IC2602 \#6 & F5 & $1.95_{-0.41}^{+0.68}$ & $52.80_{-0.05}^{+0.05}$ & 29.92 & $1.31_{-0.09}^{+0.31}$ & $52.21_{-0.06}^{+0.05}$ & 29.24 \\
\hline IC2602 \#48 & F6 & $3.28_{-0.42}^{+0.55}$ & $53.09_{-0.03}^{+0.03}$ & 30.55 & $0.94_{-0.01}^{+0.01}$ & $53.22_{-0.01}^{+0.01}$ & 30.29 \\
\hline IC2602 \#48 & F7 & $2.61_{-0.46}^{+0.67}$ & $53.04_{-0.05}^{+0.05}$ & 30.53 & $0.94_{-0.01}^{+0.01}$ & $53.22_{-0.01}^{+0.01}$ & 30.29 \\
\hline
\end{tabular}

Table 4 Loop lengths of flaring loops derived by using rise and decay method $\left(L_{r}\right)$ and Haisch's approach $\left(L_{H a}\right)$. Maximum pressure $(\mathrm{p})$, plasma density $\left(\mathrm{n}_{\mathrm{e}}\right)$, loop volume $(\mathrm{V})$, minimum magnetic field $(\mathrm{B})$ to confine the plasma and heating rate per unit volume at the flare peak $\left(\mathrm{E}_{\mathrm{h}}\right)$ are also estimated.

\begin{tabular}{|c|c|c|c|c|c|c|c|c|c|}
\hline Cluster ID & Flare & $\begin{array}{l}\mathrm{T}_{\max }^{\mathrm{r}} \\
\mathrm{MK}\end{array}$ & $\begin{array}{l}\mathrm{L}_{\mathrm{r}} \\
\left(10^{10}\right) \\
\mathrm{cm}\end{array}$ & $\begin{array}{l}\mathrm{L}_{\mathrm{Ha}} \\
\left(10^{10}\right) \\
\mathrm{cm} \\
\end{array}$ & $\begin{array}{l}\mathrm{p} \\
\left(10^{3}\right) \\
\text { dyne } \mathrm{cm}^{-2}\end{array}$ & $\begin{array}{l}\mathrm{n}_{\mathrm{e}} \\
\left(10^{11}\right) \\
\mathrm{cm}^{-3}\end{array}$ & $\begin{array}{l}\mathrm{V} \\
\left(10^{30}\right) \\
\mathrm{cm}^{3}\end{array}$ & $\begin{array}{l}\mathrm{B} \\
\left(10^{3}\right) \\
\mathrm{G}\end{array}$ & $\begin{array}{l}\mathrm{E}_{\mathrm{h}} \\
\operatorname{erg~s}^{-1} \mathrm{~cm}^{-3}\end{array}$ \\
\hline \multicolumn{10}{|c|}{$\begin{array}{l}\text { Intermediate mass star } \\
\end{array}$} \\
\hline NGC $869 \# 67$ & F1 & $179_{-83}^{+891}$ & $3.83_{-1.66}^{+7.65}$ & $5.51_{-1.22}^{+1.25}$ & 54.3 & 11.0 & 3.0 & 1.2 & 52.0 \\
\hline NGC $869 \# 140$ & $\mathrm{~F} 2$ & $>42$ & $>1.27$ & $3.42_{-1.06}^{+1.09}$ & 2.2 & 1.9 & 63.1 & 0.2 & 3.1 \\
\hline \multicolumn{10}{|c|}{ Low mass stars } \\
\hline NGC $869 \# 42$ & F3 & $34^{+49}$ & $2.93^{+2.67}$ & $9.84^{+1.71}$ & 0.5 & 0.5 & 570.2 & 0.1 & 0.3 \\
\hline NGC 869 \#111 & $\mathrm{F} 4$ & $208_{-83}^{+281}$ & $3.78_{-1.55}^{+3.37}$ & $8.41_{-2.08}^{+2.03}$ & 87.5 & 15.2 & 1.3 & 1.5 & 91.7 \\
\hline IC $2602 \# 6$ & F5 & $44_{-11}^{+18}$ & $2.10_{-0.77}^{+1.05}$ & $4.92_{-1.35}^{+1.31}$ & 1.5 & 1.2 & 4.2 & 0.2 & 1.3 \\
\hline IC $2602 \# 48$ & F6 & $81_{-12}^{+16}$ & $3.04_{-0.56}^{+0.68}$ & $3.50_{-0.30}^{+0.30}$ & 6.3 & 2.8 & 1.5 & 0.4 & 5.1 \\
\hline IC $2602 \# 48$ & F7 & $62_{-13}^{+19}$ & $3.81_{-1.96}^{+2.41}$ & $\begin{array}{l}1.79_{-1.20}^{+1.03} \\
1.03\end{array}$ & 3.1 & 1.8 & 3.4 & 0.3 & 2.4 \\
\hline
\end{tabular}

